\title{
High specificity PCR screening for 22q11.2 microdeletion in three different ethnic groups
}

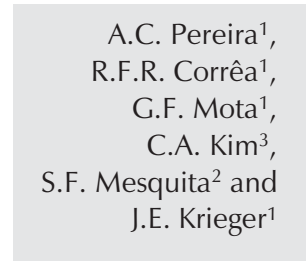

\author{
${ }^{1}$ Laboratório de Genética e Cardiologia Molecular, and ${ }^{2}$ Cardiologia Pediátrica e \\ Unidade de Doenças Cardiológicas Congênitas em Adulto, Instituto do Coração, \\ Faculdade de Medicina, Universidade de São Paulo, São Paulo, SP, Brasil \\ ${ }^{3}$ Unidade de Genética Clínica, Departamento de Pediatria, Faculdade de Medicina, \\ Universidade de São Paulo, São Paulo, SP, Brasil
}

\section{Correspondence}

J.E. Krieger

Laboratório de Genética e

Cardiologia Molecular

InCor, HC, FM, USP

Av. Dr. Eneas C. Aguiar, 44

05403-000 São Paulo, SP

Brasil

Fax: +55-11-3069-5068

E-mail: krieger@incor.usp.br

Research supported by FAPESP

(No. 95-4668-6), CNPq (No. 520696) and FINEP (No. 66.9300.2300).

Received December 2, 2002 Accepted August 18, 2003

\begin{abstract}
Congenital heart defects are the most common of all human birth defects. Numerous studies have shown that a deletion within chromosome 22q11 is associated with DiGeorge syndrome and certain forms of sporadic congenital cardiovascular disease. We have determined the value of a PCR assay using markers D22S941, D22S944 and D22S264 designed for the screening of 22q11.2 deletion through consecutive homozygosity in an ethnically admixed urban population. The study population comprised 149 unrelated men and women from three different ethnic groups (white, mulatto and black). Test specificity for the overall population was estimated at $98.3 \%$. We found no significant difference when comparing heterozygosity indices and ethnicity $(\mathrm{P}$ value $=0.43(\mathrm{D} 22 \mathrm{~S} 944), 0.22(\mathrm{D} 22 \mathrm{~S} 264)$, and 0.58 (D22S941)). There was no significant difference regarding assay specificity between the three different ethnic groups studied. This assay could constitute a cost-effective way to screen a large number of patients at increased risk, since PCR techniques are easily available, are fast, can be automatized, and are significantly less expensive than fluorescence in situ hybridization.
\end{abstract}

Key words - DiGeorge syndrome - PCR screening - $22 q 11.2$

\section{Introduction}

DiGeorge syndrome was first described in 1968 by DiGeorge, who documented thymic hypoplasia in association with hypoparathyroidism (1). In 1981, de la Chapelle et al. (2) reported a chromosome 20;22 translocation in four affected members from the same family. Another name for the collective abnormalities occurring in this syndrome has been CATCH22 (3) or the CATCH phe- notype (4) (from: cardiac defects, abnormal facies, thymic hypoplasia, cleft palate, hypocalcemia, and chromosome 22).

A deletion within chromosome 22q11 has been identified in the majority of patients with DiGeorge syndrome, velocardiofacial syndrome, conotruncal anomaly face (or Takao syndrome) and in some cases of Cayler cardiofacial syndrome and Opitz G/BBB syn- 
drome (5). In addition, several studies have shown that a deletion within chromosome $22 \mathrm{q} 11$ is associated with certain forms of sporadic congenital cardiovascular disease, including tetralogy of Fallot with and without pulmonary atresia, truncus arteriosus, and interruption of the aortic arch (6-8). In fact, substantial clinical heterogeneity has been observed with respect to the cardiovascular phenotypes associated with deletion of chromosome $22 \mathrm{q} 11$, ranging from complex intracardiac and aortic arch anomalies to completely normal anatomy (9).

It should also be emphasized that many patients with isolated congenital heart disease present symptoms as neonates or young infants, while the same may not necessarily be true for other phenotypic manifestations of the syndrome. In particular, facial features may not be apparent in infants, renal findings are not evident upon routine examination (10), and the characteristic speech and learning difficulties typically do not become clear until beyond infancy (11). Therefore, an early diagnosis of a 22q11 deletion in infants with congenital cardiovascular disease allows for early detection of associated noncardiac features, as well as appropriate genetic counseling $(9,12)$. These findings broaden the classical indications for testing, warranting screening for 22q11.2 deletion in infants with sporadic congenital heart disease as well.

Ninety percent of patients with $22 \mathrm{q} 11$ deletion syndrome (22q11DS) have a deletion of approximately 3 million base pairs, while $7 \%$ have smaller deletions of 1.5 million bases (13). Similar to other syndromes caused by a microdeletion, the molecular diagnosis of $22 \mathrm{qDS}$ is usually made by fluorescence in situ hybridization (FISH). Although FISH is considered to be the "gold standard" for the molecular diagnosis of 22q11DS, some inconveniences may arise from its use as the sole methodology for the diagnosis.

In particular, cost may preclude the use of FISH as a means of screening large numbers of candidate patients, especially in places where cytogenetics facilities are not easily available. PCR assays based on homozygosity at consecutive markers in the DiGeorge chromosomal region have been developed to circumvent these problems (14). However, no information on the use of this screening tool is available for admixed populations in which allele frequencies may vary according to ethnicity.

The aim of our study was to develop a PCR assay that could determine the deletion status at the DiGeorge syndrome locus in a cost-effective manner. In addition, we investigated if the assay sensitivity varied for different ethnic subgroups in an admixed urban area.

\section{Material and Methods}

\section{Study population}

The study population comprised 149 unrelated men and women admitted to the Blood Donation Center of the Fundação PróSangue, São Paulo University Medical School. The study was approved by the Hospital Ethics Committee and written informed consent was obtained from all participants.

The population was divided into three groups according to gender and ethnic morphological criteria. Group I included 50 white subjects, group II 50 mulatto subjects, and group III 49 black subjects. Mulatto, in this study, is defined as an individual with a known family history of admixture between black and white populations. The ethnic morphological subgroup classification was based on phenotype: pigmentation of the abdomen, hair color, type and conformation of the nose and lips, and family history, as determined and agreed upon by two examining physicians (15). It should be noted that our subgroup stratification was based on morphological criteria and not on true ethnic group stratification. Ethnic group stratifica- 
tion is defined by a group of genetic, linguistic and cultural characteristics. In particular, it has been shown that in the Brazilian population morphological criteria are not highly concordant with ancestry $(16,17)$. Venous blood was obtained for genomic DNA extraction.

\section{2q11.2 polymorphic marker genotyping}

We selected three different highly polymorphic markers contained in the 22q11.2 commonly microdeleted region. These markers are D22S944, D22S264 and D22S941. Their position relative to the studied genomic region is depicted in Figure 1. Primers for specific amplification of the polymorphic regions were as follow: D22S941 (F - CAG GTT ACA AAG TAC ATT AAC TT; R CAA GAA ATG GTT GGA GCT GGT); D22S944 (F - CAT GTG AAA GAT GCT ACT TCC; R - ATC CCA TGC TCC TCC CCA T); D22S264 (F - ATT AAC TCA TAA AGG AGC CC; R - CAC CCC ACC AGA GGT ATT CC). PCR were performed with $50 \mathrm{ng}$ of genomic DNA in a total volume of $25 \mu 1$ containing $10 \mathrm{pmol}$ of each primer, 4 nmol dNTPs, $0.5 \mathrm{U}$ Taq polymerase (Pharmacia, Uppsala, Sweden) in $1 \mathrm{x}$ $\left(\mathrm{NH}_{4}\right)_{2} \mathrm{SO}_{4}$ buffer $\left(16 \mathrm{mmol} / 1\left[\mathrm{NH}_{4}\right]_{2} \mathrm{SO}_{4}, 67\right.$ $\mathrm{mmol} / \mathrm{l}$ Tris, $\mathrm{pH} 8.8,0.01 \%$ Tween), and 1.5 $\mathrm{mmol} / 1 \mathrm{MgCl}_{2}$. PCR conditions were as follows: initial denaturation at $94^{\circ} \mathrm{C}$ for $5 \mathrm{~min}$, followed by 30 cycles of denaturation $\left(94^{\circ} \mathrm{C}\right.$ for $30 \mathrm{~s}$ ), annealing $\left(60^{\circ} \mathrm{C}\right.$ for $\left.30 \mathrm{~s}\right)$, and extension $\left(72^{\circ} \mathrm{C}\right.$ for $\left.30 \mathrm{~s}\right)$. The PCR products were visualized by electrophoresis on $7.5 \%$ polyacrylamide gel, and later stained with SYBR green I dye and stored in digital form after image acquisition with the Storm System (Molecular Dynamics Inc., Sunnyvale, CA, USA). The staining protocols were those described by Stothard et al. (18).

\section{Statistical analysis}

Alleles were defined according to PCR product size. Allele and genotype frequencies were defined by allele counting. Heterozygosity index was defined as the chance of finding an individual from a particular population heterozygous for a marker and was estimated for a marker for a locus with " $n$ " alleles by the formula: $\mathrm{H}$ (or Het) $=1$ $\left\{\left[(\text { frequency of allele } 1)^{2}\right]+[(\right.$ frequency of allele 2$\left.\left.)^{2}\right]+\ldots+\left[(\text { frequency of allele } n)^{2}\right]\right\}$. Differences in heterozygosity index were determined by the chi-square test. Linkage disequilibrium statistics, haplotype frequency estimation and test of population differentiation were conducted using the Arlequin software (19). A P value less than 0.05 was considered significant. Test specificity was defined as [ 1 - (chance of homozygosity of $n$ consecutive markers)].

\section{Results}

The markers assayed in relation to the most common site of microdeletion are shown in Figure 1. Allele frequencies for each of the studied groups are presented in Table 1.

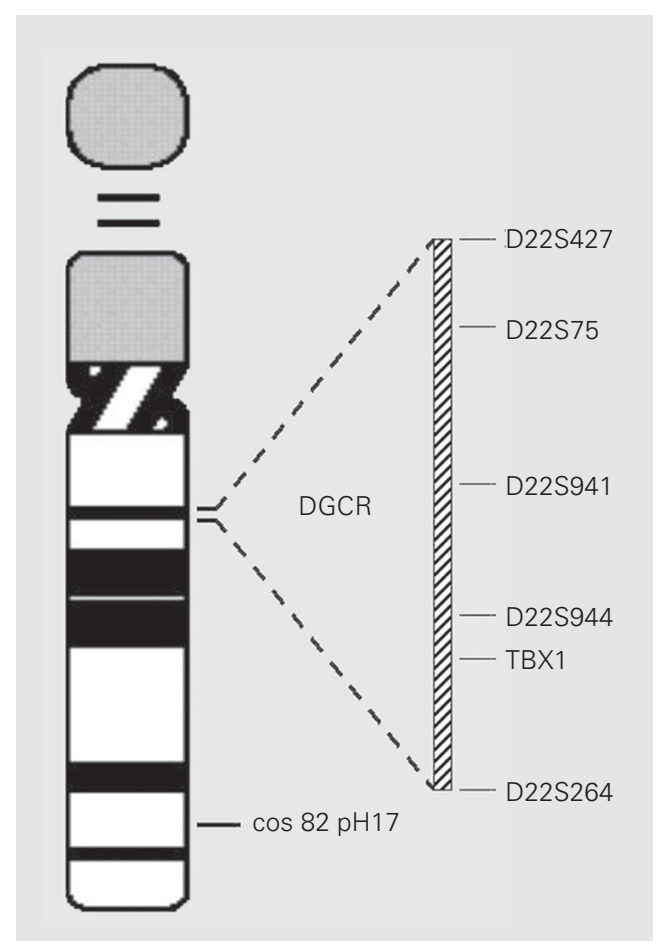

Figure 1. Idiogram of chromosome 22 illustrating the commonly deleted region [DiGeorge syndrome chromosome region (DGCR); hatched box], the relative location of the tested markers, and the location of the human T-box gene (TBX1). 
No significant difference was observed between samples in the exact test of sample differentiation based on haplotype frequencies either for a global test among all samples $(\mathrm{P}=0.08)$ or between all pairs of samples (Caucasian versus Mulatto: $\mathrm{P}=0.24$, Cauca-

\begin{tabular}{lcccc}
\multicolumn{5}{l}{ Table 1. Allele frequencies of the studied markers. } \\
\hline Allele & Population & White & Mulatto & Black \\
\hline D22S944 & & & & \\
1 & 6.7 & 8 & 10 & 2 \\
2 & 36.3 & 53 & 31 & 24.6 \\
3 & 26.5 & 18 & 29 & 32.5 \\
4 & 28.8 & 21 & 29 & 36.8 \\
5 & 1.7 & 0 & 1 & 4.1 \\
D22S264 & & & & \\
1 & 19.7 & 23 & 24 & 11.2 \\
2 & 6 & 10 & 6 & 2 \\
3 & 23.1 & 25 & 26 & 18.4 \\
4 & 22.8 & 27 & 20 & 21.5 \\
5 & 20.4 & 15 & 20 & 26.5 \\
6 & 8 & 0 & 4 & \\
D22S941 & & & & 28.4 \\
1 & 18.4 & 11 & 16 & 14.3 \\
2 & 22.5 & 27 & 26 & 26.6 \\
3 & 43.6 & 53 & 51 & 4 \\
4 & 14.1 & 9 & 7 & \\
5 & 1.4 & 0 & 0 & \\
\hline
\end{tabular}

Numbers are reported in percent.

Table 2. Sample heterozygosity index.

\begin{tabular}{lcccc}
\hline Allele & Overall & White & Mulatto & Black \\
\hline D22S944 & 70.8 & 63.6 & 72.6 & 69.9 \\
D22S264 & 80.4 & 77.9 & 79 & 79.5 \\
D22S941 & 70.4 & 62.6 & 64.2 & 75.5 \\
Specificity & 98.3 & 97 & 97.9 & 98.5
\end{tabular}

Figure 2. Assay specificity in relation to the number of markers assayed. Note that specificity does not increase significantly after addition of a fourth marker to the test.

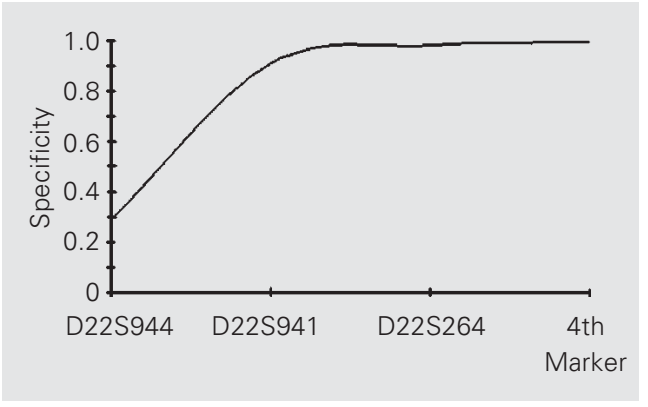

sian versus Black: $\mathrm{P}=0.09$, and Mulatto versus Black: $\mathrm{P}=0.32$ ).

In addition, the only statistically significant pairwise linkage disequilibrium observed was between markers D22S944 and D22S264 in the mulatto sample ( $\mathrm{P}=0.008)$. All other pairwise comparisons in the three studied ethnic groups were nonsignificant for linkage disequilibrium.

Heterozygosity indices are shown in Table 2. No statistical difference was found when comparing heterozygosity indices and ethnicity ( $\mathrm{P}$ value $=0.43$ (D22S944), 0.22 (D22S264), and 0.58 (D22S941)).

Assay specificity was calculated based on the heterozygosity indices described for the overall population. By using only the heterozygosity index for the given alleles, test specificity was estimated for our overall population to be $98.3 \%$ if only the proband was assayed (i.e., the risk patient). There was no significant difference regarding assay specificity between the three different ethnic groups studied. However, it is important to stress that no allele specification was used in these calculations.

In Figure 2 we present data showing how specificity was increased by using three polymorphic markers. It is interesting to note that by adding a fourth marker to the system (with a heterozygosity of $72.5 \%$ ) specificity increased by only $5 \%$.

\section{Discussion}

Congenital heart defects are the most common of all human birth defects and are the leading cause of death in the first year of life. Congenital heart defects involving the outflow tract of the heart and the vessels arising from it are due to abnormal development of neural crest-derived cells that populate the heart. The branchial arches, which give rise to the thymus, and the parathyroid glands are also populated by neural crest cells. Approximately $90 \%$ of individuals with cardiac and craniofacial defects have mono- 
allelic microdeletion of chromosome 22q11.2.

Goldmuntz et al. (6), in a study designed to determine the frequency of the 22q11 deletion in a large, prospectively ascertained sample of 251 patients with conotruncal defects, identified the deletion in $17.9 \%$ of the patients. The deletion frequency varied with the primary diagnosis and was highest in patients with interrupted aortic arch and truncus arteriosus (50 and $34.5 \%$, respectively).

Also, McElhinney et al. (9), by studying 66 patients known to have abnormalities of branching or laterality of the aortic arch without associated intracardiac defects or interrupted aortic arch, identified a 22q11 deletion in $24 \%$ of these patients. In particular, a 22q11 deletion was present in $14 \%$ of patients with a double aortic arch, $22 \%$ of patients with a right aortic arch and mirrorimage branching of the brachiocephalic vessels, $32 \%$ of patients with a right aortic arch and aberrant left subclavian artery, and 29\% of patients with a left aortic arch and aberrant right subclavian artery.

It is clear from these studies that infants with congenital heart disease, especially of conotroncus origin, should be tested for 22 q11.2 chromosomal deletion. This fact is even more relevant if one takes into account the potential benefit of preventive measures that could be applied if such a diagnosis were defined (12).

However, molecular diagnosis of 22q11.2 deletion is not widely available. It is of paramount importance, therefore, to make methodologies available that could guarantee screening of a large number of individuals at tertiary care centers. Our objective here was to determine heterozygosities using a PCR assay designed for the screening of 22q11.2 deletion through consecutive homozygosity. In addition, proposed PCR-based screening methods have not been systematically tested in different ethnic populations. Since differences in allele frequencies is the rule among different populations, it is to be expected that different test specificities may jeopar- dize the use of such screening method for a given ethnic group of an admixed population.

Our data established the allele and genotype distribution of three different polymorphic markers located in the DiGeorge syndrome chromosomal region. In addition, heterozygosity of the markers in the studied groups has defined this assay specificity to be approximately $98 \%$ in the general population. It is interesting to note that adding a fourth marker to the assay, which would increase costs, would not significantly change the specificity of the assay. Finally, we did not find statistically significant differences regarding ethnicity in marker heterozygosity indices. This fact is of clinical importance, since assay specificity will not be significantly different despite the ethnic origin of the proband.

An important aspect that should be taken into account was that the specificity estimates provided here were derived from the heterozygosity indices of the studied markers, and no allele specification was used in these calculations. Although the overall allelic content of the populations studied did not differ significantly, as shown by the exact test of population differentiation, if one analyzes some particular alleles, significant differences in their distribution may be detected among the studied ethnic groups. The use of this information when applying a diagnosis algorithm may add some information for the interpretation of the positive predictive value of a positive test, since more common alleles may present homozygosity at a higher frequency in a particular ethnic group. However, the use of allele typing information would require increasing sophistication in assay development (use of an allele calling system, i.e., molecular size markers and allele typing standards), rather than just the identification of heterozygosity for a particular marker. In addition, one would never obtain $100 \%$ specificity so as to eliminate the need for a confirmatory test (FISH 
test or loss of heterozygosity using parental DNA samples).

Understanding the etiology of DiGeorge syndrome has been confounded by several observations including the fact that deletion size does not correlate with phenotype. Carlson et al. (13) have determined that approximately $90 \%$ of velocardiofacial syndrome patients have a $3-\mathrm{Mb}$ chromosomal deletion in 22q11.2. Also, in their study all patients with the common 3-Mb deletion had the same proximal and distal chromosomal breakpoints. The markers tested in our population are included in this $3-\mathrm{Mb}$ region. In addition, a small proportion of the patients have deletions that have the same proximal breakpoint as seen for the $3-\mathrm{Mb}$ deletion, but have a nested distal deletion chromosomal breakpoint resulting in a deletion that is estimated to be $1.5 \mathrm{Mb}$. In this second group of patients, rearrangement occurs between the D22S944 and D22S264 markers. In this scenario the specificity of our test is reduced since only two markers are contained in the deleted region. This should not constitute a major drawback for the use of the assay since in these rare situations (estimated to occur in less than $7 \%$ of velocardiofacial syndrome patients) the use of parental DNA can define loss of heterozygosity.

Recently, three different reports have proposed the T-box gene (TBX1) as a candidate for the cause of congenital heart defects in DiGeorge syndrome (20-22). Thus, it was also important to define the TBX1 chromosomal region in relation to our markers. As shown in Figure 1, human TBX1 is localized between markers D22S944 and D22S264. Deletions within this region are assayed by our test.

The assay described here could constitute a cost-effective way to screen a large number of patients at increased risk since PCR techniques are more often available, are fast, can be automatized, and are significantly less expensive than FISH studies. This is also the first time that heterozygosity for the studied markers was assessed in an admixed population. It is important to emphasize, however, that these observations cannot be readily extended to other highly mixed urban populations worldwide, in which ethnicity can still be an issue when considering markers and test specificity.

\section{References}

1. Kirkpatrick Jr JA \& DiGeorge AM (1968). Congenital absence of the thymus. American Journal of Roentgenology, Radium Therapy, and Nuclear Medicine, 103: 32-37.

2. de la Chapelle A, Herva R, Koivisto M \& Aula P (1981). A deletion in chromosome 22 can cause DiGeorge syndrome. Human Genetics, 57: 253-256

3. Wilson DI, Burn J, Scambler P \& Goodship J (1993). DiGeorge syndrome, part of CATCH 22. Journal of Medical Genetics, 30: 852856.

4. Burn J (1999). Closing time for CATCH22. Journal of Medical Genetics, 36: 737-738.

5. Online Mendelian Inheritance in Man (OMIM)TM. Johns Hopkins University, Baltimore, MD, USA. MIM Number: 188400. [http:// www.ncbi.nlm.nih.gov/omim/]. Accessed January 4, 2003.

6. Goldmuntz E, Clark BJ, Mitchell LE et al. (1998). Frequency of 22q11 deletions in patients with conotruncal defects. Journal of the American College of Cardiology, 32: 492-498.

7. Chessa M, Butera G, Bonhoeffer P, Iserin L, Kachaner J, Lyonnet S, Munnich A, Sidi D \& Bonnet D (1998). Relation of genotype 22q11 deletion to phenotype of pulmonary vessels in tetralogy of Fallot and pulmonary atresia-ventricular septal defect. Heart, 79: 186-190.
8. Amati $F$, Mari A, Digilio MC, Mingarelli R, Marino B, Giannotti A, Novelli G \& Dallapiccola B (1995). 22q11 deletions in isolated and syndromic patients with tetralogy of Fallot. Human Genetics, 95: 479-482.

9. McElhinney DB, Clark 3rd BJ, Weinberg PM, Kenton ML, McDonaldMcGinn D, Driscoll DA, Zackai EH \& Goldmuntz E (2001). Association of chromosome 22q11 deletion with isolated anomalies of aortic arch laterality and branching. Journal of the American College of Cardiology, 37: 2114-2119.

10. Goodship J, Robson SC, Sturgiss S, Cross IE \& Wright C (1997). Renal abnormalities on obstetric ultrasound as a presentation of DiGeorge syndrome. Prenatal Diagnosis, 17: 867-870.

11. Gerdes M, Solot C, Wang PP et al. (1999). Cognitive and behavior profile of preschool children with chromosome 22q11.2 deletion. American Journal of Medical Genetics, 85: 127-133.

12. Gerdes M, Solot C, Wang PP, McDonald-McGinn DM \& Zackai EH (2001). Taking advantage of early diagnosis: preschool children with the 22q11.2 deletion. Genetics in Medicine, 3: 40-44.

13. Carlson C, Sirotkin H, Pandirta R et al. (1997). Molecular definition of $22 q 11$ deletions in 151 velo-cardio-facial syndrome patients. American Journal of Human Genetics, 61: 620-629. 
14. Driscoll DA, Emanuel BS, Mitchell LE \& Budarf ML (1997). PCR assay for screening patients at risk for 22q11.2 deletion. Genetic Testing, 1: 109-113.

15. Krieger $\mathrm{H}$, Morton NE, Mi MP, Azevedo E, Freire-Maia A \& Yasuda N (1965). Racial admixture in north-eastern Brazil. Annals of Human Genetics, 29: 113-125.

16. Alves-Silva J, da Silva Santos M, Guimarães PE, Ferreira AC, Bandelt HJ, Pena SD \& Prado VF (2000). The ancestry of Brazilian mtDNA lineages. American Journal of Human Genetics, 67: 444-461.

17. Carvalho-Silva DR, Santos FR, Rocha J \& Pena SDJ (2001). The phylogeography of Brazilian Y-chromosome lineages. American Journal of Human Genetics, 68: 281-286.

18. Stothard JR, Frame IA \& Miles MA (1997). An evaluation of four staining methods for the detection of DNA in nondenaturing poly- acrylamide gels. Annals of Biochemistry, 253: 262-264.

19. Schneider S, Roessli D \& Excoffier L (2000). Arlequin Ver. 2000: A Software for Population Genetics Data Analysis. Genetics and Biometry Laboratory, University of Geneva, Switzerland.

20. Merscher S, Funke B, Epstein JA et al. (2001). TBX1 is responsible for cardiovascular defects in velo-cardio-facial/DiGeorge syndrome. Cell, 104: 619-629.

21. Jerome LA \& Papaioannou VE (2001). DiGeorge syndrome phenotype in mice mutant for the T-box gene, TBX1. Nature Genetics, 27 286-291.

22. Lindsay EA, Vitelli F, Su H et al. (2001). Tbx1 haploinsufficiency in the DiGeorge syndrome region causes aortic arch defects in mice. Nature, 410: 97-101. 